\title{
Aortic Sinus of Valsalva Aneurysm Isolation by Radiofrequency Ablation in Outflow Tract Ventricular Tachycardia
}

\author{
Nicholas Olson, Scott W. Ferreira, Peter C. Mikolajczak, Ali Mehdirad \\ Division of Electrophysiology, Saint Louis University, Saint Louis, USA \\ Email: nolson02@gmail.com
}

Received 8 February 2014; revised 12 March 2014; accepted 20 March 2014

Copyright $(\underset{2014}{2}$ by authors and Scientific Research Publishing Inc.

This work is licensed under the Creative Commons Attribution International License (CC BY). http://creativecommons.org/licenses/by/4.0/

(c) (i) Open Access

\begin{abstract}
Idiopathic monomorphic ventricular tachycardia and premature ventricular complexes (PVCs) commonly arise from the right and left ventricular outflow tracts (VOT). Their mechanism is most commonly triggered activity from delayed after-depolarizations and successful ablation is performed at the site of earliest endocardial activation. Re-entrant mechanisms have been rarely described. We report a case of an otherwise healthy patient who ultimately underwent six electrophysiology studies (EPS) and suffered numerous implantable cardiac defibrillator (ICD) discharges prior to the successful radiofrequency ablation (RFA) of two idiopathic VOT tachycardias. During the sixth EPS, a proximal aortogram demonstrated a left aortic sinus of valsalva (LASV) aneurysm. Subsequntly, a novel and successful RFA strategy of aneurysm isolation was undertaken. The presence of multiple clinical or inducible VT morphologies and the characterization of a VT as re-entrant should raise concerns that a complex arrhythmogenic substrate is present and defining the anatomy with angiography or an alternative imaging modality is essential in achieving a successful ablation strategy.
\end{abstract}

\section{Keywords}

Ventricular Tachycardia, Outflow Tract, Aortic Cusp, Sinus of Valsalva, Aneurysm, Normal Heart, Radiofrequency Ablation, Re-Entry

\section{Introduction}

Sustained monomorphic ventricular tachycardia (SMMVT) is an arrhythmia that occurs almost exclusively in adolescents and young adults without structural heart disease. The mechanism of this arrhythmia has classically 
been attributed to triggered activity originating from the ventricular outflow tracts and management with radiofrequency ablation targeting the site of earliest endocardial activation is first line therapy [1] [2]. Less commonly, however, alternative mechanisms and subtle structural abnormalities can play a role. We hereby describe a case of re-entrant SMMVT in a young adult associated with an aortic sinus cusp aneurysm and a novel RF ablation strategy utilized to cure the patient.

\section{Case}

A 34 years old male presented to an outlying emergency room in October 1995 with palpitations, dyspnea, and lightheadedness. An ECG revealed sustained monomorphic ventricular tachycardia at 230 beats/min with a left bundle branch block (LBBB) morphology, inferior axis, R wave transition in V3, and negative QRS complexes in lead 1, AVL and AVR (VT1, Figure 1). After failed attempts at pharmacologic cardioversion with lidocaine and procainamide, normal sinus rhythm was restored with a 200 joule synchronized cardioversion. An EPS was performed and the clinical tachycardia was induced with triple ventricular extra-stimuli. Coronary angiography, exercise testing and a cardiac MRI revealed normal coronary arteries, no exercise induced arrhythmias, and normal cardiac structure and function respectively. Repeat EPS despite a similar pacing protocol with and without isoproterenol infusion, failed to induce ventricular tachycardia. The patient subsequently underwent abdominal ICD implantation and experienced appropriate ICD discharges from 1995 to 2004.

After suffering an ICD discharge in April 2004, the patient elected to undergo a repeat EPS at an outside hospital. Programmed stimulation induced a 300 msec cycle length SMMVT with a left bundle branch block morphology, inferior axis, R wave transition in V4, positive QRS complexes in lead 1 and AVL, and a negative QRS complexes in AVR (VT2, Figure 2). This tachycardia was clearly different from the patient's original characterized VT (VT1). Activation mapping with a basket catheter deployed in the RVOT yielded earliest endocardial activation (20 msec prior to QRs onset) in an anterior location. Pace mapping in sinus rhythm yielded a near identical match at this location and RF ablation was reported as successful. No subsequent VT occurred, a fourth EPS failed to induce a ventricular tachycardia and, therefore, the patient's ICD was explanted in June 2004.

In February 2006, the patient returned to the emergency department with palpitations and a 12 lead ECG demonstrating sustained VT1. After cardioversion the patient was taken to the EP lab for his fifth EP study with the intent to map and ablate VT1. Decremental pacing from the RV apex while on isoproterenol induced VT1. Activation mapping with the CARTO electroanatomic mapping system (Biosense Webster, Diamond Bar, CA) demonstrated a broad area of early activation (35 msec prior to QRS onset) $0.5 \mathrm{~cm}-1 \mathrm{~cm}$ below the aortic valve along the aorto-mitral continuity. The RVOT, coronary sinus, and aortic sinuses did not demonstrate early activation. Bipolar pacing from the RVOT and LVOT did not fulfill criteria for manifest entrainment. The arrhythmia could not be terminated with burst pacing.

Twelve mg and $18 \mathrm{mg}$ boluses of adenosine transiently lengthened the underlying atrial cycle length but had no effect on the VT cycle length. RFA lesions delivered at the earliest site of endocardial activation along the aorto-mitral continuity as described above (12 lesions for a total of 2 minutes and 37 seconds via a Biosense Webster $4 \mathrm{~mm}$ tip, non-irrigated, D-curve, 60 degree Celsius temperature limited ablation catheter at 50 watts) had no effect on the tachycardia. The patient was subsequently sedated and cardioverted with a 200 joule synchronized shock. Bipolar pace mapping in the area of earliest endocardial activation was performed with no match in QRS morphology. At this point, while still on an isoproterenol drip, spontaneous VT1 recurred, requiring a second 200 joule shock. After discussing the results of the EP study with the patient once awake, it was decided to give a trial of more aggressive beta blocker therapy.

Within months, the patient experienced a recurrence of VT1 requiring cardioversion at an outside hospital. During a sixth EPS, the aortic sinuses were more thoroughly mapped. In this study, a $4 \mathrm{~mm}$ tip, non-irrigated, 60 degree Celsius temperature limited ablation catheter was advanced deep into the left aortic sinus of valsalva (LASV). Fractionated electrograms extending into early diastole were noted during sinus rhythm (Figure 3(a)). Pacing at this site during sinus rhythm elicited intermittent capture beats with 2 distinct QRS morphologies with exact 12/12 matches for VT1 and VT2. Decremental pacing from this site was able to induce both VT1 and VT2. Following induction of VT2, fractionated electrograms were recorded spanning diastole (Figure 3(b)). After defining the ostium of the left main coronary artery with a Judkins left 6F catheter (Cordis, Bridgewater, NJ) 1 $1.5 \mathrm{~cm}$ above this area of interest in the LASV, RF ablation was attempted. However, RF power was severely limited to ( $<10$ Watts) secondary to high temperatures. Despite the power limit, VT was terminated during RF 


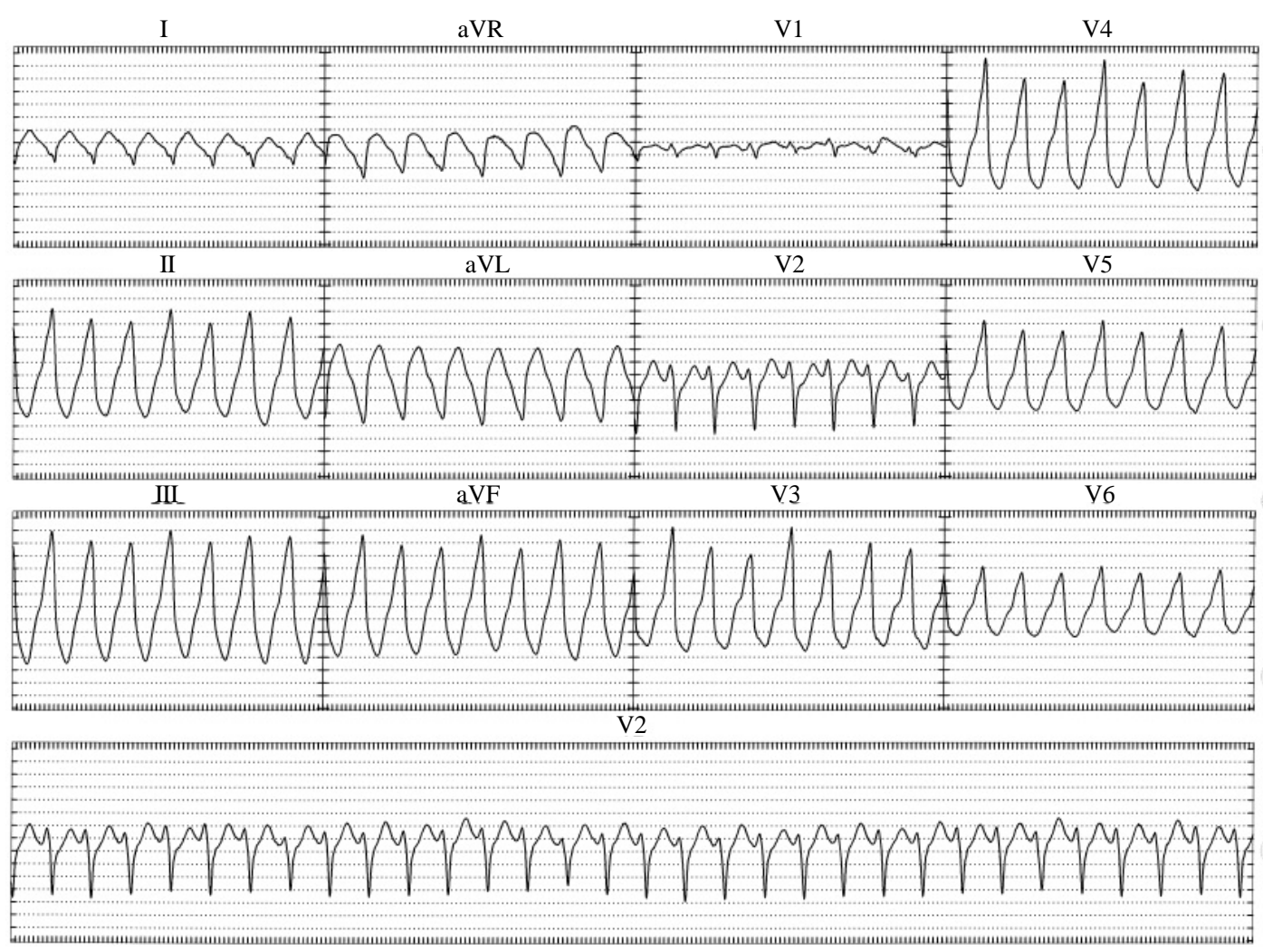

Figure 1. Ventricular Tachycardia 1 (VT1).

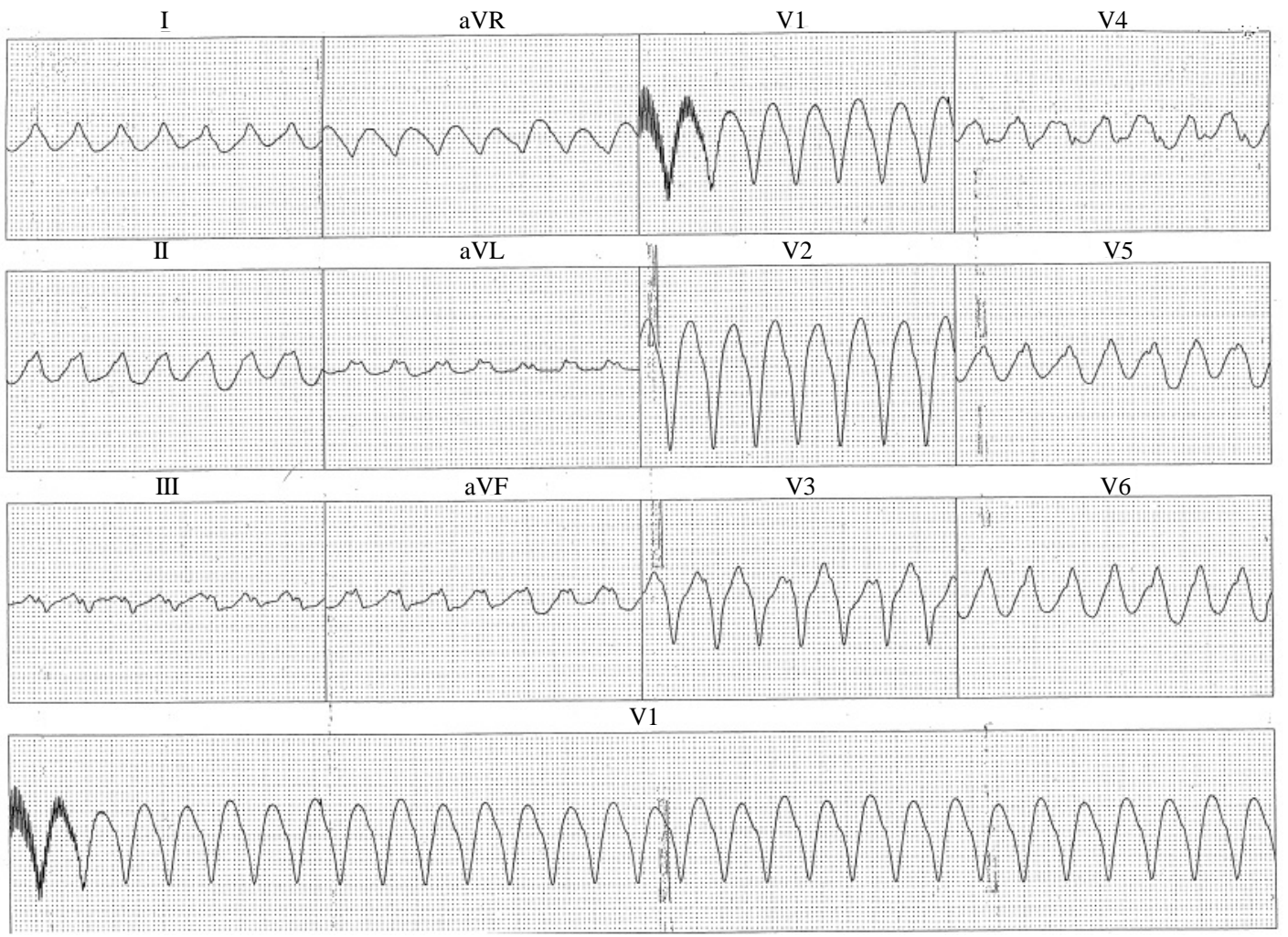

Figure 2. Ventricular Tachycardia 2 (VT2). 
application on 3 separate occasions. However, decremental pacing was able to re-induce both VT1 and VT2. Subsequently, a limited proximal aortogram was performed using the Judkins left catheter revealing a small LASV aneurysm (Figure 4). The ablation catheter was placed into the aneurysm again revealing fractionated electrograms extending into mid diastole during sinus rhythm. Pacing elicited capture beats with QRS morphologies matching VT1 and VT2. Ablation within the aneurysm was not possible due to power limitation and an unacceptably high risk of aneurysm perforation. Thus a strategy of aneurysm isolation by RFA from the LASC was pursued. A circumferential RF lesion set was delivered around the orifice of the aneurysm in a point by point manner. Power was titrated up to 40 Watts with a temperature limit of 50 degrees Celsius. Individual lesions were created with 20 seconds of RF delivery. After electrical isolation of the aneurysm, further attempts to re-initiate the ventricular tachycardias with and without isoproterenol and aggressive pacing were unsuccessful. Subsequent imaging studies, including TEE and a cardiac gated CT scan, have demonstrated a stable nonenlarging LASV aneurysm. The patient has remained arrhythmia free 7 years post ablation without anti-arrhythmic or beta blocker therapy.

\section{Discussion}

The mechanism of the majority of outflow tract ventricular tachycardias is due to increased intracellular cAMP levels and abnormal calcium handling, resulting in delayed after-depolarizations and consequent triggered activity [1]-[4]. However, re-entrant mechanisms have also been implicated. Nakano et al. performed electrophysiological testing on 51 consecutive patients referred for evaluation of idiopathic outflow tract ventricular tachycardia [5]. Six patients were determined to have VT of re-entrant mechanism via entrainment mapping. A critical isthmus was most reliably identified and ablated in either the left or right aortic sinus. Reithmann et al. described a patient with a well-tolerated left bundle branch block (LBBB), inferior axis ventricular tachycardia who underwent electrophysiology testing and ablation from the right side of the septum [6]. In addition to the

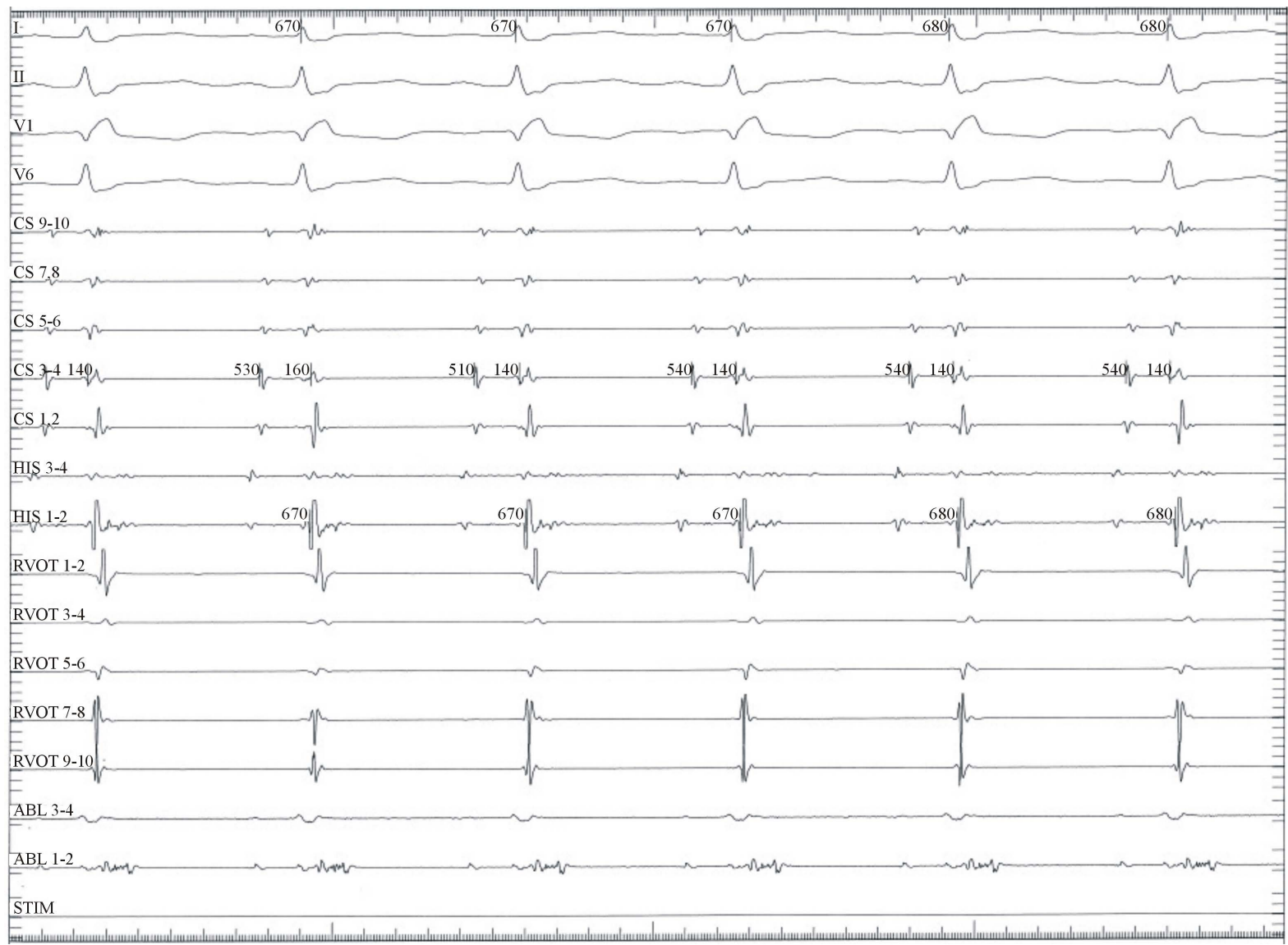

(a) 


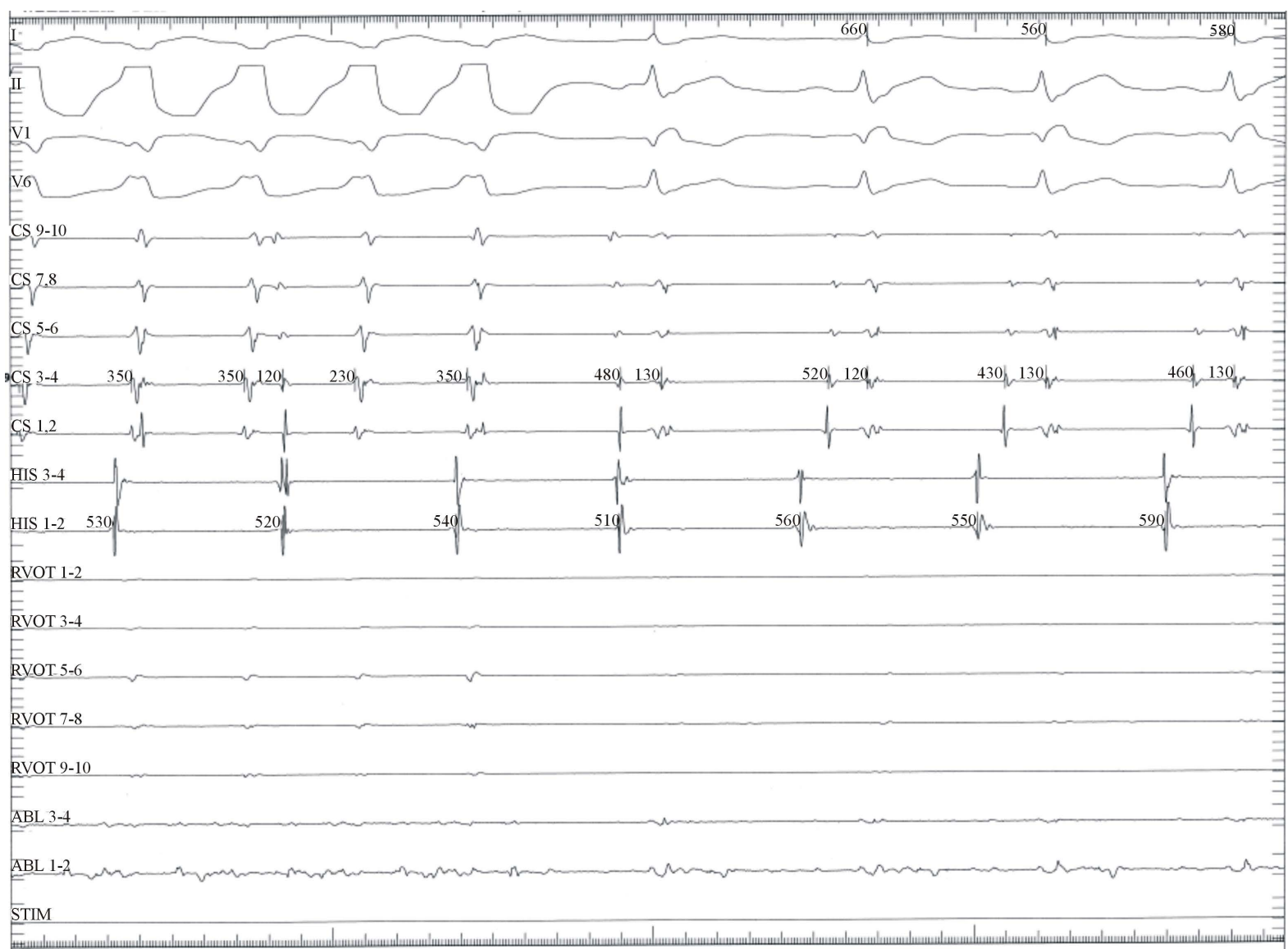

(b)

Figure 3. (a) Intracardiac tracing from deep within the left coronary aortic cusp during sinus rhythm demonstrating a fractionated electrogram extending into early diastole. RVOT: deca-polar catheter placed along the anterior RVOT extending distally to below the pulmonary valve. CS: deca-polar coronary sinus catheter. His: quadrapolar His catheter. Abl: ablation catheter within the left coronary cusp aneurysm. (b) Intracardiac tracing from within the left coronary aortic cusp aneurysm during VT2 demonstrating a fractionated electrogram spanning the entire diastolic interval. Once the ventricular tachycardia breaks fractionated electrograms can be seen spanning into mid-disatole. RVOT: deca-polar catheter placed along the anterior RVOT extending distally to below the pulmonary valve. CS: deca-polar coronary sinus catheter. His: quadrapolar His catheter (not in place). Abl: ablation catheter within the left coronary cusp aneurysm.

LBBB, inferior axis VT a second VT with right bundle branch block (RBBB) and inferior axis was noted clinically and induced by programed stimulation. Activation mapping of the RVOT during the LBBB tachycardia revealed a mid-diastolic potential and pacing from this site revealed concealed entrainment with a stimulus to QRS of 100 - 150 msec. After ablation at this site, neither the LBBB nor the RBBB, inferior axis ventricular tachycardia could be induced. Yamada et al. described a patient with an inferior axis, LBBB VT that was induced via programmed stimulation [7]. Earliest activation was noted in the right coronary cusp with a very long stimulus to QRS delay (85 msec). Notably, a similar paced morphology was noted in the basal anterior LV epicardium with a short stimulus to QRS (40 msec). Concealed conduction was likely occurring from the right coronary cusp to an epicardial breakthrough site via a slowly conducting, electrically isolated myocardial fiber. This fiber may also have provided a substrate for re-entry. Another case published by Yamada et al. described a patient with an inferior axis VT with QRS alternans (beat to beat alternation of ventricular activation/QRS morphology) [8]. Earliest activation was again noted in the right coronary cusp and pacing from that site yielded a morphology match with reproducible QRS alternans. Ablation in the right coronary cusp successfully eradicated both VT morphologies. Again, electrically isolated myocardial fibers in the outflow tract region were likely at play, resulting in displacement of earliest ventricular activation from the area of earliest activation. Whether these fibers created a substrate for re-entry or were simply by-stander pathways in the setting of an alternative 


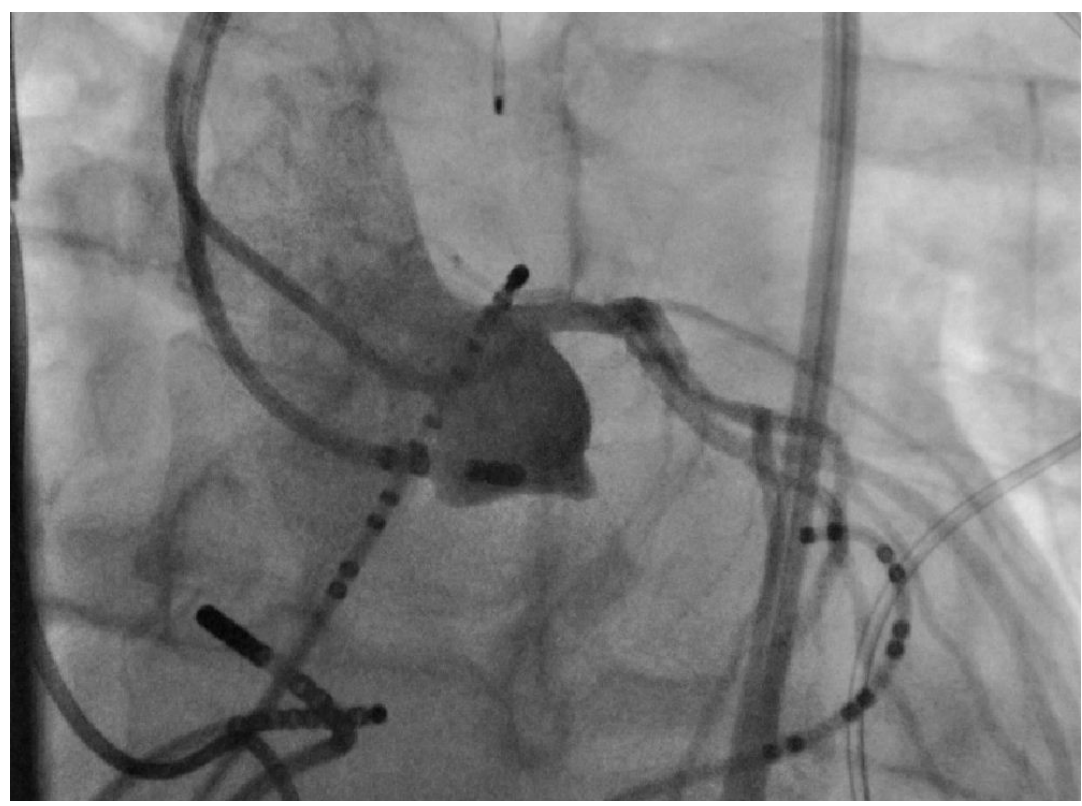

Figure 4. Proximal aortogram in the left anterior oblique projection demonstrating a small LASV aneurysm.

underlying tachycardia mechanism is unclear.

Although outflow tract VT is commonly referred to as "normal heart ventricular tachycardia" because of the uniformly preserved biventricular size and function, subtle structural abnormalities such as congenital outflow tract, basal LV, or aortic sinus of valsalva aneurysms have been described and implicated in arrhythmia pathogenesis [9]-[12]. In the case previously described by Reithman et al., a LV basal aneurysm was identified in the area adjacent to the critical septal isthmus utilized by both tachycardias [6]. In a case by Patel et al., repeated attempts at pulmonary artery catheterization resulted in salvos of ventricular tachycardia [13]. Transesophageal echocardiography revealed an aortic sinus of valsalva aneurysm protruding into the RVOT precluding catheter advancement. A more recent case report by Chadha et al. described a patient who presented with SMMVT (inferior axis, LBBB morphology, negative QRS in leads V1-V5) [14]. A transthoracic echocardiogram revealed an $8 \times 7 \mathrm{~cm}$ right aortic sinus cusp aneurysm that was causing compression of the right ventricular outflow tract. Often, anatomic abnormalities are only identified by imaging studies performed after failed catheter ablation.

\section{Conclusion}

Our case is the first to describe ventricular tachycardia originating from an aortic sinus cusp aneurysm successfully treated with aneurysm isolation by RFA. The mechanism of the ventricular tachycardia was likely re-entrant with VT1 and VT2 representing either multiple exit sites from a common isthmus, two distinct isolated isthmuses, or bi-directional propagation across a common isthmus. The tachycardia was induced with decremental pacing and fractionated electrograms were recorded within the aneurysm extending into early diastole during sinus rhythm and throughout diastole during ventricular tachycardia. Adenosine was ineffective in terminating the arrhythmia or prolonging the tachycardia cycle length. Manifest entrainment could not be demonstrated from the RV apex or the RV outflow tract. However, this is not surprising considering the presumably small re-entrant circuit and isolated isthmus within the left aortic sinus cusp aneurysm. The case is novel and clinically important for two reasons. First, although rare, outflow tract VT can be associated with subtle structural abnormalities. The presence of multiple VT morphologies (either seen clinically or induced in the EP lab) or characterization as a re-entrant mechanism should raise concern that a complex arrhythmogenic substrate is present and technical difficulty with RFA will be more likely. In this setting, defining the local anatomy with angiography or an alternative imaging modality is essential in performing a successful ablation. In our case, it was only with the 6th EP study that the proximal aortogram was taken and the left aortic sinus cusp aneurysm was identified. Secondly, this case demonstrates the novel use of arrhythmogenic substrate isolation in treating 
outflow tract VT. Such a methodology has potential application whenever catheter accessibility is limited or where direct RF application is deemed too risky. In our case, a continuous line of RF ablation lesions was placed around the orifice of the left aortic sinus cusp aneurysm. The focal origin of the VT or the VT's isolated isthmus, in the case of a re-entrant mechanism, was electrically isolated within the aneurysm. Our patient has remained arrhythmia free for the last 7 years without anti-arrhythmic or beta-blocker therapy.

\section{References}

[1] Lerman, B.B., Belardinelli, L., West, G.A., et al. (1986) Adenosine-Sensitive Ventricular Tachycardia: Evidence Suggesting Cyclic AMP-Mediated Triggered Activity. Circulation, 74, 270-280. http://dx.doi.org/10.1161/01.CIR.74.2.270

[2] Lerman, D., Stein, K., Englestein, E., et al. (1995) Mechanism of Repetitive Monomorphic Ventricular Tachycardia. Circulation, 92, 421-429. http://dx.doi.org/10.1161/01.CIR.92.3.421

[3] Farzaneh-Far, A. and Lerman, B. (2005) Idiopathic Ventricular Outflow Tract Tachycardia. Heart, 91, 136-138. http://dx.doi.org/10.1136/hrt.2004.033795

[4] Lerman, B., Belardinelli, L., West, G.A., et al. (1986) Adenosine-Sensitive Ventricular Tachycardia: Evidence Suggesting Cyclic AMP-Mediated Triggered Activity. Circulation, 74, 270-280. http://dx.doi.org/10.1161/01.CIR.74.2.270

[5] Nakano, E., Harada, T., Aonuma, K., et al. (2012) Identification of Unusual Reentry Circuit Sites of Nonischemic Ventricular Outflow Tract Tachycardia. Journal of Cardiovascular Electrophysiology, 23, 179-187. http://dx.doi.org/10.1111/j.1540-8167.2011.02149.x

[6] Reithmann, C., Remp, T. and Steinbeck, G. (2006) Substrate in the Interventricular Septum for Left Ventricular and Right Ventricular Outflow Tract Tachycardia: Ablation from the Right Side of the Septum. Journal of Cardiovascular Electrophysiology, 17, 321-324. http://dx.doi.org/10.1111/j.1540-8167.2005.00311.x

[7] Yamada, T., McElderry, H.T., Doppalapudi, H., et al. (2008) Ventricular Tachycardia with a Myocardial Fibre Travelling from the Origin in the Right Aortic Sinus Cusp to the Epicardial Breakout Site of the Right Ventricular Outflow Tract. Europace, 10, 469-470. http://dx.doi.org/10.1093/europace/eun051

[8] Yamada, T., McElderry, H.T., Doppalapudi, H., et al. (2010) QRS Alternans During Idiopathic Ventricular Tachycardia Originating from the Right Aortic Cusp of the Aorta. Europace, 12, 1335.

http://dx.doi.org/10.1093/europace/eup326

[9] Channer, K., Hutter, J. and George, M. (1988) Unruptured Aneurysm of the Sinus of Valsalva Presenting with Ventricular Tachycardia. European Heart Journal, 9, 186-190.

[10] Farand, P., Brochu, M., Gervais, A., et al. (2009) Familial Unruptured Sinus of Valsalva Aneurysm Obstructing the Right Ventricular Outflow Tract. Canadian Journal of Cardiology, 25, 227-228. http://dx.doi.org/10.1016/S0828-282X(09)70072-8

[11] Vijayalakshmi, I.B., Devanada, N.S. and Chitra, N.A. (2009) Patient with Aneurysms of Both Aortic Coronary Sinuses of Valsalva Obstructing Both Ventricular Outflow Tracts. Cardiology in the Young, 19, 537-539. http://dx.doi.org/10.1017/S1047951109990710

[12] Thankachen, R., Gnanamuthu, R., Doshi, H., et al. (2003) Unruptured Aneurysm of the Sinus of Valsalva Presenting with Right Ventricular Outflow Obstruction. Texas Heart Institute Journal, 30, 152-154.

[13] Patel, C. and Baily, P.L. (2006) Sinus of Valsalva Aneurysm and Ventricular Tachycardia during Pulmonary Artery Catheterization. Anesthesia \& Analgesia, 102, 369-371. http://dx.doi.org/10.1213/01.ane.0000189255.30188.cC

[14] Chadra, S., Lodha, A., Shetty, V., et al. (2012) Sinus of Valsalva Aneurysm: A Rare Presentation with Ventricular Tachycardia. Journal of the American College of Cardiology, 59, 1729. http://dx.doi.org/10.1016/j.jacc.2011.11.065 